\title{
Rare Case of Primary Hemophagocytic Lymphohistiocytosis: A Case Report Authors
}

\author{
Dr Anurita Saigal $^{1^{*}}$, Dr Anchana Gulati ${ }^{2}$, Dr Sudarshan Kumar Sharma ${ }^{3}$ \\ Dr Rajni Kaushik ${ }^{4}$
}

${ }^{1}$ Junior Resident of Pathology, ${ }^{2}$ Associate Professor of Pathology, ${ }^{3}$ Professor and HOD Department of

Pathology, ${ }^{4}$ Professor of Pathology

Indira Gandhi Medical College, Shimla, Himachal Pradesh, India

*Corresponding Author

Dr Anurita Saigal

Department of Pathology, Indira Gandhi Medical College, Shimla, Himachal Pradesh, India

Email: anuritasaigal@gmail.com

\begin{abstract}
Hemophagocytic lymphohistiocytosis $(H L H)$ is a fatal disease in which uncontrolled activation of immune system occurs. It is characterized by fever, pancytopenia, splenomegaly and hemophagocytosis in bone marrow, liver or lymph nodes. It has been associated with viral, bacterial, fungal and parasitic infection. An early diagnosis and treatment is the key to decrease the mortality. We report a case of hemophagocytic lymphohistiocytosis in a 39 year old male patient presenting with prolonged fever, pancytopenia, hepatosplenomegaly.

Keywords: Hemophagocytic lymphohistiocytosis; Macrophage, immune activation.
\end{abstract}

\section{Introduction}

Hemophagocytic lymphohistiocytosis (HLH) is a life threatening hematological disorder characterized by over activation of $\mathrm{T}$ lymphocytes and macrophages leading to uncontrolled over production of inflammatory cytokines with resultant multi organ inflammation. HLH can be acquired or familial; it has different etiologies, including but not limited to malignancies, autoimmune disorders as well as infections. ${ }^{1}$ In comparison to children, it is difficult to diagnose adults with HLH since it occurs with a variety of different diseases and most of the literature on HLH is derived from a pediatric population. We present a case of a Haemophagocytic syndrome in a 39 year old male patient.

\section{Case Report}

39 year old male presented with two month history of progressive malaise, body aches, fever without chills and rigor. On per abdomen examination-splenomegaly $4 \mathrm{~cm}$ below costal margin was present. Complete Haemogram revealed pancytopenia. Haemoglobin was 6.9 $\mathrm{gm} / \mathrm{dl}$, Platelet count was $57000 / \mathrm{mm} 3$, Total leukocyte count was $1900 / \mathrm{mm}^{3}$.Peripheral blood examination revealed Macrocytic anaemia with thrombocytopenia. In view of Pancytopenia, his bone marrow examination (aspiration and biopsy), was done. 


\begin{tabular}{|c|l|}
\hline BIOCHEMICAL PROFILE & \multicolumn{1}{c|}{ LEVELS } \\
\hline Bilirubin & Raised $(1.79 \mathrm{mg} / \mathrm{dl})$ \\
\hline Ferritin & Raised $(12892 \mathrm{ng} / \mathrm{ml})$ \\
\hline LDH & Raised $(1057 \mathrm{u} / \mathrm{l})$ \\
\hline Triglyceride & Raised $(>265 \mathrm{mg} / \mathrm{dl})$ \\
\hline
\end{tabular}

Bone marrow aspiration was a Dry tap.

Imprint smears focally revealed histiocytes showing haemophagocytosis.(Figure1)

On bone marrow biopsy haemopoietic tissue was replaced by sheets of histiocytes. (Figure 2)

On Immunohistochemistry Cells were CD68 (Figure 3) and CD163 positive but negative for CD1a and Langerin negative excluding Langerhans cell histiocytosis ( $\mathrm{LCH}$ ) which comes as an important differential. Soluble IL-2 receptor (sCD25) were elevated.

As per the revised criteria, five of the eight criteria are required to fulfill a clinical diagnosis of HLH, although patients with a molecular diagnosis, that is, one of the known FHL mutations, do not necessarily need to fulfill the diagnostic criteria ${ }^{1}$.

The diagnosis of HLH can be established if either 1 or 2 below are fulfilled.

A molecular diagnosis consistent with HLH.

Diagnostic criteria for HLH fulfilled (5 out of 8 criteria below):

Fever

Splenomegaly

Cytopenia affecting $=2$ of 3 lineages:

Hemoglobin $<90 \mathrm{~g} / \mathrm{L}$, Platelets $<100 \quad \times 10^{9} / \mathrm{L}$; Neutrophils $<1.0 \times 10^{9} / \mathrm{L}$

Hypertriglyceridemia and/ or hypofibrinogenemia: fasting triglycerides $=3.0 \mathrm{mmol} / \mathrm{L}$

$(=265 \mathrm{mg} / \mathrm{dl})$, OR fibrinogen $=1.5 \mathrm{~g} / \mathrm{L}$

Hemophagocytosis in bone marrow, spleen or lymph nodes

Low or absent NK-cell activity (using local laboratory reference ranges)

Ferritin $=500 \mathrm{ug} / \mathrm{L}$

Soluble CD25 (i.e., soluble IL-2 receptor) $=2,400$ $\mathrm{U} / \mathrm{m}$

Diagnostic criteria for HLH were fulfilled according to Revised Diagnostic Guidelines for hemophagocytic lymphohistiocytosis (HLH).
After considering all the above findings, diagnosis of Haemophagogocytic lymphohistiocytosis was given. The patient died within 4 months of diagnosis.

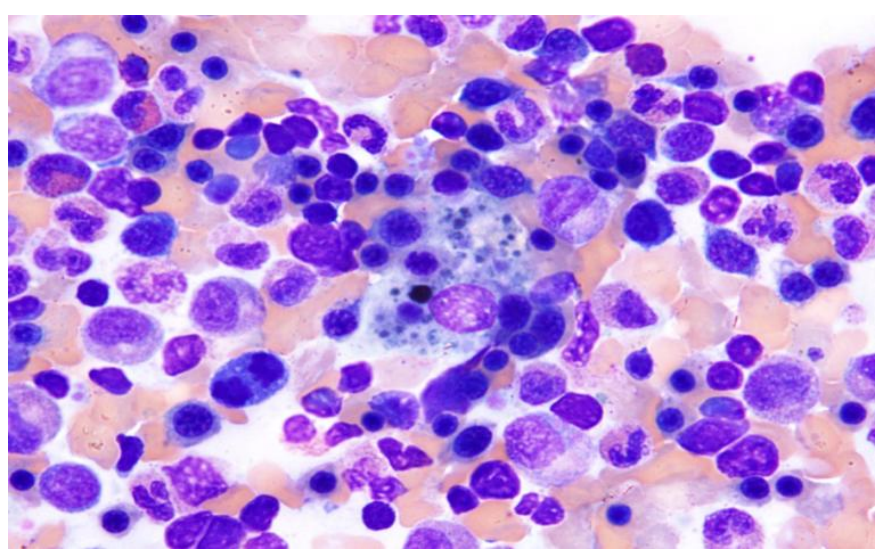

Figure 1-Imprint smear reveals histiocyte showing phagocytosis of platelet, lymphocyte and erythroid cell (H \& E 400X)

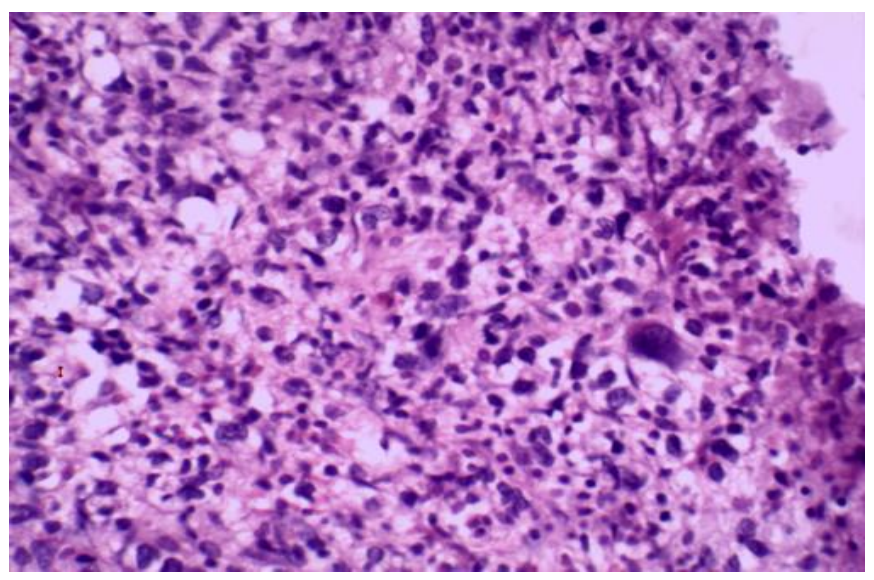

Figure 2-On bone marrow biopsy haemopoietic tissue was replaced by sheets of histiocytes $(\mathrm{H} \&$ E stain, 400X)

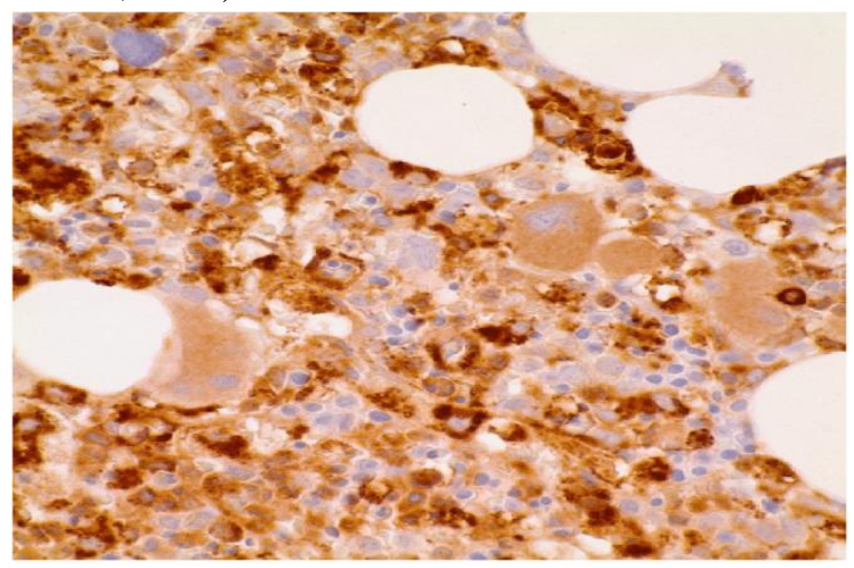

Figure 3-Bone Marrow biopsy showing CD68 positive macrophages engulfing nucleated red blood cells 


\section{Discussion}

Hemophagocytic lymphohistiocytosis (HLH) is a clinical entity characterized by a sustained activation of the mononuclear phagocytic system that may result in an extreme hyper

inflammatory response ${ }^{2}$. Criteria for the diagnosis of HLH proposed by the Histiocyte Society ${ }^{4}$ include clinical, laboratory, and histopathologic features. Fever and splenomegaly are the most common clinical signs, but hepatomegaly, lymphadenopathy, jaundice, and rash are also seen. In this case 39 year old male presented with fever, malaise, splenomegaly associated with Cytopenias and raised biochemical markers.

HLH can occur in two forms: genetic and acquired. Genetic HLH is further categorized into a familial form (familial hemophagocytic lymphohistiocytosis [FHLH]) and is associated with immune deficiencies such as ChédiakHigashi syndrome (CHS). In most cases, HLH is the only manifestation of the familial variant Secondary HLH is also known as acquired HLH and commonly presents in adulthood. It is usually associated with an underlying infection, malignancy or an autoimmune disease which can lead to a hyperactive immune response ${ }^{3}$. HLH associated with infection may be difficult to distinguish from familial HLH triggered by an infection. The distinction is important; as allogeneic bone marrow transplantation is the therapy of choice in patients with familial $\mathrm{HLH}^{5}$. Sporadic HLH has a better prognosis. HLH triggered by bacterial infection is associated with a high recovery rate.

Histopathologically hemophagocytosis is seen in bone marrow, spleen, and lymph nodes and occasionally the central nervous system and skin. Activated macrophages may engulf erythrocytes, leukocytes, and platelets, their precursors, and cellular fragments.LCH is an important diagnosis was ruled out in this case by IHC (negative CD1a).

Appropriate broad spectrum antibiotics and supportive therapy are given. Early recognition and treatment with chemotherapeutic agents or bone marrow transplant may reduce mortality.

\section{Conclusion}

HLH is a clinical syndrome of hyper inflammation, and uncontrolled and ineffective immune response. A high level of suspicion for HLH is required in patients presenting with splenomegaly, cytopenias ,elevated liver markers and increased inflammatory markers.HLH can be rapidly progressive and potentially fatal if left undiagnosed and undertreated. Early detection and treatment may decrease the morbidity and mortality of patients.

\section{References}

1. Janka GE. Familial and acquired hemophagocytic lymphohistiocytosis. Annu Rev Med. 2012

2. Otrock ZK, Eby CS: Clinical characteristics, prognostic factors, and outcomes of adult patients with hemophagocytic lymphohistiocytosis. Am J Hematol. 2014， 90:220224. 10.1002/ajh.23911

3. Nikiforow $\mathrm{S}$, Berliner $\mathrm{N}$ : The unique aspects of presentation and diagnosis of hemophagocytic lymphohistiocytosis in adults. Hematology Am Soc Hematol Educ Program. 2015, 2015:183189. 10.1182/asheducation-2015.1.183

4. Risma K, Jordan MB. Hemophagocytic lymphohistiocytosis updates and evolving concepts. Curr Opin Pediatr. 2012;24(1):9-15.

5. Henter JI, Horne A, Arcio M, et al, HLH 2004,Diagnostic and therapeutic guidelines for Hemophagocytic Lymphohistiocytosis. Pediatric Blood Cancer 2007;48:124-131. 